\title{
Helices on a surface in Euclidean 3- space
}

\author{
Ufuk Öztürk ${ }^{1 *}$, Hasan Hilmi Hacısalihoğlu² \\ ${ }^{1}$ Çankırı Karatekin University, Faculty of Science, Department of Mathematics, \\ 18100, Çankırı, Turkey, +90 (376) 218 9537, \\ ozturkufuk06@gmail.com, uuzturk@asu.edu \\ 2 Bilecik Şeyh Edebali University, Faculty of Arts and Sciences, Department of Mathematics, \\ 11210, Bilecik, Turkey, \\ ${ }^{2}$ Current Address: Taşpınar Mahallesi 2856. Sokak No:14, Ahlatlıbel, Gölbaşı, Ankara, \\ +90 535492 9030, h.hacisali@science.ankara.edu.tr \\ * Corresponding author \\ Received: $28^{\text {th }}$ August 2016 \\ Accepted: $4^{\text {th }}$ December 2016 \\ DOI: http://dx.doi.org/10.18466/cbujos.302650
}

\begin{abstract}
In this paper, we define the strip slant helices according to the frame of the strip and introduce some characterizations for strip slant helices using the curvatures of the strip. We also determine the axis of the strip slant helices. Moreover, we investigate some characterizations for the strip slant helices when the curve of the strip is a geodesic curve or an asymptotic curve or a principal curve.
\end{abstract}

Keywords - Geodesic curvature, geodesic torsion, helix, normal curvature, strip

\section{Introduction}

Some of the classical results of differential geometry topics in Riemannian geometry have been treated by the researchers. Several authors introduced different types of helices and give some characterizations of these special curves for a long time. The helix is generally known as a curve in DNA double and $\alpha$-form. Also we can see the helix curve in the field of computer aided design and computer graphics. In differential geometry; it is well-known that a general helix (or a curve of constant slope) is a curve whose tangent's makes a constant angle with a fixed direction, which is called the axis of the helix. The ratio of the curvature and the torsion of such curve is a constant, which is the necessary and sufficient condition for a curve to be a general helix [1].

In [2] izumiya Izumiya and Takeuchi introduced a slant helix as a curve in the Euclidean 3-space having a property that its principal normal vector makes a constant angle with a constant direction (see also [3]) and in [4] Kula et al. consider the tangent spherical indicatrix (the normal and binormal indicatrix, respectively) and characterize slant helices by certain differential equations verified for each one of these indicatrices. Moreover, in [5] Ali and López generalize the definition of slant helices in the Euclidean fourdimensional space $\mathbb{E}^{4}$, and present different characterizations of them. Recently, in [6] Ali and Turgut give some characterizations of slant helices in the $n$-dimensional Euclidean space. Moreover, they introduce the type- 2 harmonic curvatures of a regular curve.

In differential geometry of surfaces, a strip or curvesurface pair is a natural moving frame constructed along the curve $\alpha$ on a surface and it is the analog of the Frenet-Serret frame. On the strip in Euclidean space have studied in [7,8]. In [7] Hacisalihoglu studied a relation between the Serret-Frenet formulae of a curve $\alpha$ in a hypersurface $M$ and the curvatures of $M$ in 
CBÜ F Bil. Dergi., Cilt 13, Sayı 1, 2017, 113-123 s

Euclidean space $\mathbb{E}^{n}$. In [8] Sabuncuoglu and Hacisalihoglu calculated the higher curvature of a strip in $\mathbb{E}^{n}$

In this paper, we define the strip slant helices according to the frame of the strip and characterize the strip slant helices using the curvatures of the strip. We also determine the axis of the strip slant helices. Moreover, we investigate some characterizations for the strip slant helices when the curve of the strip is a geodesic curve or an asymptotic curve or a principal curve.

\section{Basic Concept}

We now recall some basic concepts on classical differential geometry of space curves and the definition of the strip in Euclidean 3-space. Let

$$
\begin{aligned}
\alpha: I \subset \mathbb{R} & \longrightarrow \mathbb{E}^{3} \\
s & \longrightarrow \alpha(s)=\left(\alpha_{1}(s), \alpha_{2}(s), \alpha_{3}(s)\right)
\end{aligned}
$$

be a curve parameterized by arc length. There exist Frenet frame $\{T, N, B\}$ at each point of $\alpha$ where $T(s)=\alpha^{\prime}(s)$ is the unit tangent vector, $N(s)=\frac{\alpha^{\prime \prime}(s)}{\left\|\alpha^{\prime \prime}(s)\right\|}$ is the principal normal vector and $B(s)=T(s) \times N(s)$ is the binormal vector field. Differentiating the Frenet frame yields the classic Frenet equations:

$$
\left[\begin{array}{l}
T^{\prime}(s) \\
N^{\prime}(s) \\
B^{\prime}(s)
\end{array}\right]=\left[\begin{array}{ccc}
0 & \kappa(s) & 0 \\
-\kappa(s) & 0 & \tau(s) \\
0 & -\tau(s) & 0
\end{array}\right]\left[\begin{array}{c}
T(s) \\
N(s) \\
B(s)
\end{array}\right]
$$

where $\kappa(s)$ and $\tau(s)$ are the curvature and the torsion of $\alpha$, respectively.

Definition 1 ([8]) Let $M$ and $\alpha$ be a surface and $a$ unite velocity curve on $M$ in $\mathbb{E}^{3}$, respectively. The locus of the surface elements of $M$, which are the part of the tangent plane of $\alpha$ at a neighborhood of every point of $\alpha$, are called a strip or curve-surface pair along the curve $\alpha$ which is showed by $(\alpha, M)$.

Let $\alpha$ be a regular unit speed curve in $\mathbb{E}^{3}$ with the Frenet frame $\{T, N, B\}$ lying fully on a regular surface $M$ and $\zeta$ be a unit normal vector field of the surface $M$ at the point $\alpha(s)$. Then, we have

$$
\zeta \times \xi=\eta,
$$

CBU J. of Sci., Volume 13, Issue 1, 2017, p 113-123

is the binormal vector field of the strip where $\xi=T$. Thus, we obtain the system of orthonormal vector fields $\{\xi(s), \eta(s), \zeta(s)\}$ is called the strip three-bundle and we have the following FrenetSerret type formulae:

$$
\left[\begin{array}{c}
\xi^{\prime}(s) \\
\eta^{\prime}(s) \\
\zeta^{\prime}(s)
\end{array}\right]=\left[\begin{array}{ccc}
0 & k_{g} & k_{n} \\
-k_{g} & 0 & \tau_{g} \\
-k_{n} & -\tau_{g} & 0
\end{array}\right]\left[\begin{array}{c}
\xi(s) \\
\eta(s) \\
\zeta(s)
\end{array}\right]
$$

Here, $k_{n}(s)=\left\langle\xi^{\prime}(s), \zeta(s)\right\rangle=\kappa \sin \theta$ is the normal curvature, $k_{g}(s)=\left\langle\xi^{\prime}(s), \eta(s)\right\rangle=\kappa \cos \theta$ is the geodesic curvature, $\tau_{g}(s)=\left\langle\eta^{\prime}(s), \zeta(s)\right\rangle=\tau-\theta^{\prime}$ is the geodesic torsion and $\theta$ is the angle between the vectors $\eta$ and $N,[7,8]$.

\section{Strip slant helices in Euclidean 3-space}

In this section, we consider a regular unit speed curve $\alpha$ on a regular surface $M$ in $\mathbb{E}^{3}$ and introduce strip slant helices according to the frame $\{\xi, \eta, \zeta\}$ of $(\alpha, M)$. We give a classisication of such curves in the Euclidean 3 -space $\mathbb{E}^{3}$. Throughout this section let $\mathbb{R}_{0}$ denotes $\mathbb{R} \backslash\{0\}$.

Definition 2 The strip $(\alpha, M)$ in $\mathbb{E}^{3}$ is called $\xi$-strip slant helix if there exists a non-zero fixed direction $U \in \mathbb{E}^{3}$ such that

$$
\langle\xi, U\rangle=\text { constant }
$$

holds. The fixed direction $U$ is called the axis of the strip slant helix.

Definition 3 The strip $(\alpha, M)$ in $\mathbb{E}^{3}$ is called $\eta$-strip slant helix if there exists a non-zero fixed direction $V \in \mathbb{E}^{3}$ such that

$$
\langle\eta, V\rangle=\text { constant }
$$

holds. The fixed direction $V$ is called the axis of the strip slant helix.

Definition 4 The strip $(\alpha, M)$ in $\mathbb{E}^{3}$ is called $\zeta$-strip slant helix if there exists a non-zero fixed direction $W \in \mathbb{E}^{3}$ such that 
CBÜ F Bil. Dergi., Cilt 13, Sayı 1, 2017, 113-123 s

$$
\langle\zeta, W\rangle=\text { constant }
$$

holds. The fixed direction $W$ is called the axis of the strip slant helix.

Let us first characterize $\xi$-type slant helices.

Case 1 ( $\xi$-strip slant helices) If $(\alpha, M)$ is $\xi$-strip slant helix parameterized by the arclength $s$ in $\mathbb{E}^{3}$, then according to Definition 2, there exists a nonzero constant vector field $U \in \mathbb{E}^{3}$ such as

$$
g(\xi, U)=c, \quad c \in \mathbb{R}_{0}
$$

ith respect to the Frenet frame $\{\xi, \eta, \zeta\}$ of $(\alpha, M)$, the fixed direction $U$ can be decomposed as

$$
U=c \xi+u_{2} \eta+u_{3} \zeta
$$

where $u_{2}$ and $u_{3}$ are differentiable functions of the curvatures. Differentiating the equation (2.5) with respect to $s$ and using equations (2.3), we obtain the following system of differential equations

$$
\left\{\begin{aligned}
u_{3} k_{n}+u_{2} k_{g} & =0 \\
u_{2}^{\prime}-u_{3} \tau_{g}+c k_{g} & =0 \\
u_{3}^{\prime}+u_{2} \tau_{g}+c k_{n} & =0
\end{aligned}\right.
$$

From the first and the second equations of (2.6) we get

$$
\left\{\begin{array}{l}
u_{2}=-c e^{-\int \frac{\tau_{g} k_{g}}{k_{n}} d s}\left(\int k_{g} e^{\int \frac{\tau_{g} k_{g}}{k_{n}} d s} d s\right), \\
u_{3}=c \frac{k_{g}}{k_{n}} e^{-\int \frac{\tau_{g} k_{g}}{k_{n}} d s}\left(\int k_{g} e^{\int \frac{\tau_{g} k_{g}}{k_{n}} d s} d s\right),
\end{array}\right.
$$

where $c \in \mathbb{R}_{0 \text {. }}$

Substituting (2.7) in the third equation of (2.6) we obtain that the curvature functions of $(\alpha, M)$ satisfy the relation

$a\left[\left(\frac{k_{g}}{k_{n}}\right)^{\prime}-\tau_{g}\left(\frac{k_{g}}{k_{n}}\right)^{2}+\tau_{g}\right]+\frac{k_{g}^{2}}{k_{n}}+k_{n}=0$,

where $a=e^{-\int \frac{\tau_{g} k_{g}}{k_{n}} d s}\left(\int k_{g} e^{\int \frac{\tau_{g} k_{g}}{k_{n}} d s} d s\right)$.

Conversely, assume that (2.8) holds. Consider the
CBU J. of Sci., Volume 13, Issue 1, 2017, p 113-123 vector $U$ given by

$$
\begin{aligned}
& U=c \xi-c e^{-\int \frac{\tau_{g} k_{g}}{k_{n}} d s}\left(\int k_{g} e^{\int \frac{\tau_{g} k_{g}}{k_{n}} d s} d s\right) \eta \\
& +c \frac{k_{g}}{k_{n}} e^{-\int \frac{\tau_{g} k_{g}}{k_{n}} d s}\left(\int k_{g} e^{\int \frac{\tau_{g} k_{g}}{k_{n}} d s} d s\right) \zeta,
\end{aligned}
$$

where $\quad c \in \mathbb{R}_{0 \text {. }}$ Differentiating the previous equation with respect to $s$ and using the equations (2.3), we find $U^{\prime}=0$. Hence $U$ is a fixed direction. It can be easily checked that

$$
g(\xi, U)=c, \quad c \in \mathbb{R}_{0}
$$

According to Definition 2, $(\alpha, M)$ is a $\xi$-strip slant helix with the axis $U$.

Therefore, we can give the following theorem and corollary.

Theorem 1 Let $(\alpha, M)$ be a strip in $\mathbb{E}^{3}$ with the curvatures $k_{g}, k_{n}$ and $\tau_{g}$. Then $(\alpha, M)$ is a $\xi$-strip slant helix if and only if its curvature functions $k_{g}, k_{n}$. and $\tau_{g}$ satisfy the relation

$$
a\left[\left(\frac{k_{g}}{k_{n}}\right)^{\prime}-\tau_{g}\left(\frac{k_{g}}{k_{n}}\right)^{2}+\tau_{g}\right]+\frac{k_{g}^{2}}{k_{n}}+k_{n}=0,
$$

where $a=e^{-\int \frac{\tau_{g} k_{g}}{k_{n}} d s}\left(\int k_{g} e^{\int \frac{\tau_{g} k_{g}}{k_{n}} d s} d s\right)$.

Corollary 1 The axis of the $\xi$-strip slant helix $(\alpha, M)$ in $\mathbb{E}^{3}$ is given by

$$
\begin{aligned}
& U=c \xi-c e^{-\int \frac{\tau_{g} k_{g}}{k_{n}} d s}\left(\int k_{g} e^{\int \frac{\tau_{g} k_{g}}{k_{n}} d s} d s\right) \eta \\
& +c \frac{k_{g}}{k_{n}} e^{-\int \frac{\tau_{g} k_{g}}{k_{n}} d s}\left(\int k_{g} e^{\int \frac{\tau_{g} k_{g}}{k_{n}} d s} d s\right) \zeta,
\end{aligned}
$$

where $c \in \mathbb{R}_{0 \text {. }}$

Substituting $c=0$ in relation (2.6), we get

$$
\begin{aligned}
& u_{2}=a_{1} e^{-\int \frac{\tau_{g} k_{g}}{k_{n}} d s}, \\
& u_{3}=-a_{1} \frac{k_{g}}{k_{n}} e^{-\int \frac{\tau_{g} k_{g}}{k_{n}} d s},
\end{aligned}
$$

where $a_{1} \in \mathbb{R}_{0}$. 
CBÜ F Bil. Dergi., Cilt 13, Sayı 1, 2017, 113-123 s

Therefore, we obtain the next corollary

Corollary 2 Let $(\alpha, M)$ be a $\xi$-strip slant helix with the axis $U$ in $\mathbb{E}^{3}$. If its tangent vector $\xi$ is orthogonal to the axis $U$, then the axis $U$ is given by

$$
U=a_{1} e^{-\int \frac{\tau_{g} k_{g}}{k_{n}} d s} \eta-a_{1} \frac{k_{g}}{k_{n}} e^{-\int \frac{\tau_{g} k_{g}}{k_{n}} d s} \zeta,
$$

where $a_{1} \in \mathbb{R}_{0}$.

Now, we consider the following subcases when the curvatures $k_{g}, k_{n}$ and $\tau_{g}$ are zero, respectively.

Case 1.1 Let the curve $\alpha$ be a geodesic curve (i. e. $\left.k_{g}=0\right)$. In this case, from (2.6) we have

$$
\left\{\begin{aligned}
u_{3} k_{n} & =0 \\
u_{2}^{\prime}-u_{3} \tau_{g} & =0 \\
u_{3}^{\prime}+u_{2} \tau_{g}+c k_{n} & =0
\end{aligned}\right.
$$

From the first equation of (2.10) we get $k_{n}=0$ or $u_{3}=0$.

(i) If $k_{n}=0$ for all $s$, then $\kappa=0$ which means

that the curve $\alpha$ is a straight line.

(ii) If $u_{3}=0$ for all $s$, then from (2.10) we get $u_{2}=-c \frac{k_{n}}{\tau_{g}} \in \mathbb{R}_{0}$. Also, since $k_{g}=\kappa \cos \theta=0$, we find that $\theta=\mp \frac{\pi}{2}$ so by using the definition of $k_{n}$ and $\tau_{g}$, we have $k_{n}=\mp \kappa$, and $\tau_{g}=\tau$. Thus, we have the frame $\{\xi, \eta, \zeta\}$ of the strip $(\alpha, M)$ as follows:

$$
\left[\begin{array}{c}
\xi^{\prime}(s) \\
\eta^{\prime}(s) \\
\zeta^{\prime}(s)
\end{array}\right]=\left[\begin{array}{ccc}
0 & 0 & \mp \kappa \\
0 & 0 & \tau \\
\pm \kappa & -\tau & 0
\end{array}\right]\left[\begin{array}{c}
\xi(s) \\
\eta(s) \\
\zeta(s)
\end{array}\right]
$$

and the axis of $(\alpha, M)$ always lies in the plane $s p\{\xi, \eta\}$ and is given by

$$
U=c \xi \pm c \frac{\kappa}{\tau} \eta
$$

where $\frac{\kappa}{\tau}=$ constant and $c \in \mathbb{R}_{0}$.
CBU J. of Sci., Volume 13, Issue 1, 2017, p 113-123 Therefore, we can give the following corollary.

Corollary 3 Let $(\alpha, M)$ be a $\xi$-strip slant helix with the axis $U$ is given by (2.11). Then the curve $\alpha$ is a geodesic curve on $M$ if only if the curve $\alpha$ is a general helix.

Case 1.2 Let the curve $\alpha$ be an asymptotic curve (i. e. $\left.k_{n}=0\right)$. In this case, from (2.6) we have

$$
\left\{\begin{aligned}
u_{2} k_{g} & =0 \\
u_{2}^{\prime}-u_{3} \tau_{g}+c k_{g} & =0 \\
u_{3}^{\prime}+u_{2} \tau_{g} & =0 .
\end{aligned}\right.
$$

From the first equation of (2.12) we get $k_{g}=0$ or $u_{2}=0$

(i) If $k_{g}=0$ for all $s$, then $\kappa=0$ which means that the curve $\alpha$ of $(\alpha, M)$ is a straight line.

(ii) If $u_{2}=0$ for all $s$, then from (2.12) we get $u_{3}=c \frac{k_{g}}{\tau_{g}} \in \mathbb{R}_{0}$. Also, since $k_{n}=\kappa \sin \theta=0$, we find that $\theta=k \pi(k=0,1)$ so by using the definition of $k_{g}$ and $\tau_{g}$, we have $k_{g}=\mp \kappa$ and $\tau_{g}=\tau$. Thus, we have the frame $\{\xi, \eta, \zeta\}$ of the strip $(\alpha, M)$ as follows:

$$
\left[\begin{array}{l}
\xi^{\prime}(s) \\
\eta^{\prime}(s) \\
\zeta^{\prime}(s)
\end{array}\right]=\left[\begin{array}{ccc}
0 & \mp \kappa & 0 \\
\pm \kappa & 0 & \tau \\
0 & -\tau & 0
\end{array}\right]\left[\begin{array}{l}
\xi(s) \\
\eta(s) \\
\zeta(s)
\end{array}\right]
$$

and the axis of $(\alpha, M)$ always lies in the plane $s p\{\xi, \zeta\}$ and is given by

$$
U=c \xi \mp c \frac{\kappa}{\tau} \zeta,
$$

where $\frac{\kappa}{\tau}=$ constant and $c \in \mathbb{R}_{0}$.

Therefore, we can give the following corollary.

Corollary 4 Let $(\alpha, M)$ be a $\xi$-strip slant helix with the axis $U$ is given by (2.13). Then the curve $\alpha$ is an asymptotic curve on $M$ if only if the curve $\alpha$ is a general helix.

Case 1.3 Let the curve $\alpha$ be a principal curve (i. e. 
CBÜ F Bil. Dergi., Cilt 13, Sayı 1, 2017, 113-123 s

$\left.\tau_{g}=0\right)$, then from (2.6) we have

$$
\left\{\begin{aligned}
u_{3} k_{n}+u_{2} k_{g} & =0 \\
u_{2}^{\prime}+c k_{g} & =0 \\
u_{3}^{\prime}+c k_{n} & =0
\end{aligned}\right.
$$

and we get

$$
\begin{aligned}
& u_{2}=-c \int k_{g} d s \\
& u_{3}=-c \int k_{n} d s
\end{aligned}
$$

Substituting (2.15) in the first equation of (2.14), we obtain that the curvature functions of $(\alpha, M)$ satisfy the relation as follows

$$
k_{g} \int k_{g} d s+k_{n} \int k_{n} d s=0
$$

Therefore, the axis of $(\alpha, M)$ is given by

$$
U=c \xi-c\left(\int k_{g} d s\right) \eta-c\left(\int k_{n} d s\right) \zeta
$$

where $c \in \mathbb{R}_{(0}$.

Therefore, we obtain the next corollary.

Corollary 5 Let $(\alpha, M)$ be a $\xi$-strip slant helix in $\mathbb{E}^{3}$. If the curve $\alpha$ is a line of principal curvature of $M$, then the axis $U$ of the $\xi$-strip slant helix $(\alpha, M)$ is

$$
U=c \xi-c\left(\int k_{g} d s\right) \eta-c\left(\int k_{n} d s\right) \zeta
$$

where $c \in \mathbb{R}_{0}$ and

$$
k_{g}\left(\int k_{g} d s\right)+k_{n}\left(\int k_{n} d s\right)=0 .
$$

Next, let us consider $\eta$-type slant helices.

Case 2 ( $\eta$-strip slant helices) If $(\alpha, M)$ is an $\eta-$ strip slant helix parameterized by the arclength $s$ in $\mathbb{E}^{3}$, then according to Definition 3 there exists a non-zero constant vector field $U \in \mathbb{E}^{3}$ such that

$$
g(\eta, V)=c, \quad c \in \mathbb{R}_{0}
$$

With respect to the Frenet frame $\{\xi, \eta, \zeta\}$ of $(\alpha, M)$, the fixed direction $V$ can be decomposed as
CBU J. of Sci., Volume 13, Issue 1, 2017, p 113-123

$$
V=v_{1} \xi+c \eta+v_{3} \zeta
$$

where $v_{1}$ and $v_{3}$ are differentiable functions of the curvatures. Differentiating the equation (2.18) with respect to $s$ and using equations (2.3), we obtain the following system of differential equations

$$
\left\{\begin{aligned}
v_{1}^{\prime}-v_{3} k_{n}-c k_{g} & =0 \\
v_{1} k_{g}-v_{3} \tau_{g} & =0 \\
v_{3}^{\prime}+v_{1} k_{n}+c \tau_{g} & =0
\end{aligned}\right.
$$

From the second and the third equations of (2.19) we get

$$
\left\{\begin{array}{l}
v_{1}=-c \frac{\tau_{g}}{k_{g}} e^{-\int \frac{\tau_{g} k_{n}}{k_{g}} d s}\left(\int \tau_{g} e^{\int \frac{\tau_{g} k_{n}}{k_{g}} d s} d s\right), \\
v_{3}=-c e^{-\int \frac{\tau_{g} k_{n}}{k_{g}} d s}\left(\int \tau_{g} e^{\int \frac{\tau_{g} k_{n}}{k_{g}} d s} d s\right),
\end{array}\right.
$$

where $c \in \mathbb{R}_{0}$.

Substituting (2.20) in the first equation of (2.19), we obtain the relation

$$
a\left[\left(\frac{\tau_{g}}{k_{g}}\right)^{\prime}-k_{n}\left(\frac{\tau_{g}}{k_{g}}\right)^{2}-k_{n}\right]+\frac{\tau_{g}^{2}}{k_{g}}+k_{g}=0,
$$

where $a=e^{-\int \frac{\tau_{g} k_{n}}{k_{g}} d s}\left(\int \tau_{g} e^{\int \frac{\tau_{g} k_{n}}{k_{g}} d s} d s\right)$.

Conversely, assume that (2.21) holds. Consider the vector $V$ given by

$$
\begin{aligned}
& V=-c \frac{\tau_{g}}{k_{g}} e^{-\int \frac{\tau_{g} k_{n}}{k_{g}} d s}\left(\int \tau_{g} e^{\int \frac{\tau_{g} k_{n}}{k_{g}} d s} d s\right) \xi \\
& +c \eta-c e^{-\int \frac{\tau_{g} k_{n}}{k_{g}} d s}\left(\int \tau_{g} e^{\int \frac{\tau_{g} k_{n}}{k_{g}} d s} d s\right) \zeta,
\end{aligned}
$$

where $\quad c \in \mathbb{R}_{0}$. Differentiating the previous equation with respect to $s$ and using the equations (2.3) and (2.19), we find $V^{\prime}=0$. Hence $V$ is a fixed direction. It can be easily checked that 
CBÜ F Bil. Dergi., Cilt 13, Sayı 1, 2017, 113-123 s

$$
g(\eta, V)=c, \quad c \in \mathbb{R}_{0} .
$$

According to Definition 3, $(\alpha, M)$ is an $\eta$-strip slant helix with the axis $V$.

Theorem 2 Let $(\alpha, M)$ be a strip in $\mathbb{E}^{3}$ with the curvatures $k_{g}, k_{n}$ and $\tau_{g}$. Then $(\alpha, M)$ is an $\eta$-strip slant helix if and only if its curvature functions $k_{g}, k_{n}$. and $\tau_{g}$ satisfy the relation

$$
\begin{aligned}
& \qquad a\left[\left(\frac{\tau_{g}}{k_{g}}\right)^{\prime}-k_{n}\left(\frac{\tau_{g}}{k_{g}}\right)^{2}-k_{n}\right]+\frac{\tau_{g}^{2}}{k_{g}}+k_{g}=0, \\
& \text { where } a=e^{-\int \frac{\tau_{g} k_{n}}{k_{g}} d s}\left(\int \tau_{g} e^{\int \frac{\tau_{g} k_{n}}{k_{g}} d s} d s\right) .
\end{aligned}
$$

Corollary 6 The axis of $\eta$-strip slant helix $(\alpha, M)$ in $\mathbb{E}^{3}$ is given by

$$
\begin{aligned}
& V=-c \frac{\tau_{g}}{k_{g}} e^{-\int \frac{\tau_{g} k_{n}}{k_{g}} d s}\left(\int \tau_{g} e^{\int \frac{\tau_{g} k_{n}}{k_{g}} d s} d s\right) \xi \\
& +c \eta-c e^{-\int \frac{\tau_{g} k_{n}}{k_{g}} d s}\left(\int \tau_{g} e^{\int \frac{\tau_{g} k_{n}}{k_{g}} d s} d s\right) \zeta,
\end{aligned}
$$

where $c \in \mathbb{R}_{0}$.

Putting $c=0$ in relation (2.19), we get

$$
\left\{\begin{aligned}
v_{1}^{\prime}-v_{3} k_{n} & =0, \\
v_{1} k_{g}-v_{3} \tau_{g} & =0, \\
v_{3}^{\prime}+v_{1} k_{n} & =0 .
\end{aligned}\right.
$$

and

$$
\begin{aligned}
& v_{1}=a_{2} \frac{\tau_{g}}{k_{g}} e^{-\int \frac{\tau_{g} k_{n}}{k_{g}} d s} \\
& v_{3}=a_{2} e^{-\int \frac{\tau_{g} k_{n}}{k_{g}} d s},
\end{aligned}
$$

where $a_{2} \in \mathbb{R}_{0}$.

Therefore, we obtain the next corollary.

Corollary 7 Let $(\alpha, M)$ be a $\eta$-strip slant helix with the axis $V$ in $\mathbb{E}^{3}$. If its binormal vector $\eta$ is orthogonal to the axis $V$, then the axis $V$ is given by
CBU J. of Sci., Volume 13, Issue 1, 2017, p 113-123

$$
V=a_{2} \frac{\tau_{g}}{k_{g}} e^{-\int \frac{\tau_{g} k_{n}}{k_{g}} d s} \xi+a_{2} e^{-\int \frac{\tau_{g} k_{n}}{k_{g}} d s} \zeta,
$$

where $a_{2} \in \mathbb{R}_{0}$.

Now, we consider the following special cases when the curvatures $k_{g}, k_{n}$ and $\tau_{g}$ are zero, respectively.

Case 2.1 Let the curve $\alpha$ is a geodesic curve (i. e. $\left.k_{g}=0\right)$, then from (2.19) we have

$$
\left\{\begin{aligned}
v_{1}^{\prime}-v_{3} k_{n} & =0 \\
v_{3} \tau_{g} & =0 \\
v_{3}^{\prime}+v_{1} k_{n}+c \tau_{g} & =0
\end{aligned}\right.
$$

From the second equation of (2.22) we get $\tau_{g}=0$ or $v_{3}=0$.

(i) If $\tau_{g}=0$ for all $s$, then from the first and the second equations of (2.22) we get

$$
\frac{d}{d s}\left(\frac{1}{k_{n}} \frac{d v_{1}}{d s}\right)+v_{1} k_{n}=0 .
$$

Putting $p(s)=\frac{1}{k_{n}(s)}$, the above equation can be rewritten as

$$
\frac{d}{d s}\left(p(s) \frac{d v_{1}}{d s}\right)+\frac{v_{1}}{p(s)}=0 .
$$

By changing the variables in the above equation by $t(s)=\int \frac{1}{p(s)} d s$, we find

$$
\frac{d^{2} v_{1}}{d t^{2}}+v_{1}=0
$$

The solution of the previous differential equation is given by

$$
v_{1}(t)=C_{1} \cos (t)+C_{2} \sin (t),
$$

and since $t(s)=\int k_{n}(s) d s$, we get

$$
v_{1}=C_{1} \cos \left(\int k_{n} d s\right)+C_{2} \sin \left(\int k_{n} d s\right) .
$$

Also, the first equation of (2.22) we have

$$
v_{3}=-C_{1} \sin \left(\int k_{n} d s\right)+C_{2} \cos \left(\int k_{n} d s\right) \text {. }
$$


On the other hand, since $k_{g}=\kappa \cos \theta=0$, we find that $\theta=\mp \frac{\pi}{2}$ so by using definition $k_{n}$ and $\tau_{g}$ we have $k_{n}=\mp \kappa$ and $\tau_{g}=\tau=0$. Therefore, the axis of $(\alpha, M)$ is given by

$$
V=v_{1} \xi+c \eta+v_{3} \zeta
$$

where

$$
\begin{aligned}
& v_{1}=C_{1} \cos \left(\int \kappa d s\right) \mp C_{2} \sin \left(\int \kappa d s\right), \\
& v_{3}=-C_{1} \sin \left(\int \kappa d s\right) \mp C_{2} \cos \left(\int \kappa d s\right) .
\end{aligned}
$$

and $\eta$-strip slant helices.

Corollary 10 Let the curve $\alpha$ be a geodesic curve on $M$. Then, $(\alpha, M)$ is a $\xi$-strip slant helix if and only if $(\alpha, M)$ is an $\eta$-strip slant helix with the axis (2.24).

Case 2.2 Let the curve $\alpha$ is an asymptotic curve (i. e. $\left.k_{n}=0\right)$, then from (2.19) we have

$$
\left\{\begin{aligned}
v_{1}^{\prime}-c k_{g} & =0, \\
v_{1} k_{g}-v_{3} \tau_{g} & =0, \\
v_{3}^{\prime}+c \tau_{g} & =0 .
\end{aligned}\right.
$$

(ii) If $v_{3}=0$ for all $s$, then from (2.22) we get $v_{1}=-c \frac{\tau_{g}}{k_{n}} \in \mathbb{R}_{0}$. Also, since $k_{g}=\kappa \cos \theta=0$, we find that $\theta=\mp \frac{\pi}{2}$ so by using the definition of $k_{n}$. and $\tau_{g}$, we have $k_{n}=\mp \kappa$ and $\tau_{g}=\tau$. Thus, we have the frame $\{\xi, \eta, \zeta\}$ of the strip $(\alpha, M)$ as follows:

$$
\left[\begin{array}{c}
\xi^{\prime}(s) \\
\eta^{\prime}(s) \\
\zeta^{\prime}(s)
\end{array}\right]=\left[\begin{array}{ccc}
0 & 0 & \mp \kappa \\
0 & 0 & \tau \\
\pm \kappa & -\tau & 0
\end{array}\right]\left[\begin{array}{c}
\xi(s) \\
\eta(s) \\
\zeta(s)
\end{array}\right]
$$

and the axis of $(\alpha, M)$ always lies on the plane $s p\{\xi, \eta\}$ and is given by

$$
V= \pm c \frac{\tau}{\kappa} \xi+c \eta
$$

where $\frac{\tau}{\kappa}=$ constant and $c \in \mathbb{R}_{0}$.

Therefore, we can give the following corollaries.

Corollary 8 Let $(\alpha, M)$ be an $\eta$-strip slant helix with the axis $V$ is given by (2.23). If the curve $\alpha$ of $(\alpha, M)$ is a geodesic curve on $M$, then the position vector of the curve $\alpha$ always lies in the plane $\operatorname{sp}\{\xi, \zeta\}$.

Corollary 9 Let $(\alpha, M)$ be an $\eta$-strip slant helix with the axis $V$ is given by (2.24). If the curve $\alpha$ of $(\alpha, M)$ is a geodesic curve on $M$, then the curve $\alpha$ is a general helix.

Also, we can give the following corollary which gives the relationship between $\xi$-strip slant helices

From the first and the third equation of (2.25), we get

$$
\begin{aligned}
& v_{1}=c \int k_{g} d s, \\
& v_{3}=-c \int \tau_{g} d s .
\end{aligned}
$$

Substituting (2.26) in the second equation of (2.25), we obtain that the curvature functions of $(\alpha, M)$ satisfy the relation as follows

$$
k_{g} \int k_{g} d s+\tau_{g} \int \tau_{g} d s=0 .
$$

Also, since $k_{n}=\kappa \sin \theta=0$, we find that $\theta=k \pi$ ( $k=0,1)$ so by using the definition of $k_{g}$ and $\tau_{g}$, we have $k_{g}= \pm \kappa$ and $\tau_{g}=\tau$. Therefore, the axis of $(\alpha, M)$ is given by

$$
V= \pm c\left(\int \kappa d s\right) \xi+c \eta-c\left(\int \tau d s\right) \zeta,
$$

where $c \in \mathbb{R}_{(0)}$. Thus, we have the frame $\{\xi, \eta, \zeta\}$ of the strip $(\alpha, M)$ as follows:

$$
\left[\begin{array}{l}
\xi^{\prime}(s) \\
\eta^{\prime}(s) \\
\zeta^{\prime}(s)
\end{array}\right]=\left[\begin{array}{ccc}
0 & \pm \kappa & 0 \\
\mp \kappa & 0 & \tau \\
0 & -\tau & 0
\end{array}\right]\left[\begin{array}{l}
\xi(s) \\
\eta(s) \\
\zeta(s)
\end{array}\right] .
$$

Case 2.3 Let the curve $\alpha$ is a principal curve (i. e. $\tau_{g}=0$ ), then from (2.19) we have 
CBÜ F Bil. Dergi., Cilt 13, Sayı 1, 2017, 113-123 s

$$
\left\{\begin{array}{r}
v_{1}^{\prime}-v_{3} k_{n}-c k_{g}=0 \\
v_{1} k_{g}=0 \\
v_{3}^{\prime}+v_{1} k_{n}=0
\end{array}\right.
$$

From the second equation of (2.28) we get $k_{g}=0$ or $u_{1}=0$.

(i) If $k_{g}=0$ for all $s$, then from the first and third equation of (2.28) we have

$$
\frac{d}{d s}\left(\frac{1}{k_{n}} \frac{d v_{1}}{d s}\right)+v_{1} k_{n}=0
$$

The solution of the previous differential equation is given by

$$
v_{1}=C_{1} \cos \left(\int k_{n} d s\right)+C_{2} \sin \left(\int k_{n} d s\right) .
$$

Also, the first equation of (2.28) we have

$$
v_{3}=-C_{1} \sin \left(\int k_{n} d s\right)+C_{2} \cos \left(\int k_{n} d s\right) .
$$

On the other hand, since $k_{g}=\kappa \cos \theta=0$, we find that $\theta=\mp \frac{\pi}{2}$ so by using the definition of $k_{n}$ and $\tau_{g}$ , we have $k_{n}=\mp \kappa$ and $\tau=0$. Therefore, the axis of $(\alpha, M)$ is given by

$$
V=v_{1} \xi+c \eta+v_{3} \zeta
$$

where

$$
\begin{aligned}
& v_{1}=C_{1} \cos \left(\int \kappa d s\right) \mp C_{2} \sin \left(\int \kappa d s\right), \\
& v_{3}=-C_{1} \sin \left(\int \kappa d s\right) \mp C_{2} \cos \left(\int \kappa d s\right) .
\end{aligned}
$$

(ii) If $v_{1}=0$ for all $s$, then from (2.28) we get $v_{3}=-c \frac{k_{g}}{k_{n}} \in \mathbb{R}_{0}$ so by the definition of $k_{n}$ and $k_{g}$ we get $\theta=\theta_{0}=$ constant. Thus, we obtain $k_{n}=\kappa \sin \theta_{0}, k_{g}=\kappa \cos \theta_{0}$ and $\tau=0$. Thus, we have the frame $\{\xi, \eta, \zeta\}$ of the strip $(\alpha, M)$ as follows:

$$
\left[\begin{array}{l}
\xi^{\prime}(s) \\
\eta^{\prime}(s) \\
\zeta^{\prime}(s)
\end{array}\right]=\kappa\left[\begin{array}{ccc}
0 & \cos \theta_{0} & \sin \theta_{0} \\
-\cos \theta_{0} & 0 & 0 \\
-\sin \theta_{0} & 0 & 0
\end{array}\right]\left[\begin{array}{c}
\xi(s) \\
\eta(s) \\
\zeta(s)
\end{array}\right]
$$

CBU J. of Sci., Volume 13, Issue 1, 2017, p 113-123 and the axis of $(\alpha, M)$ always lies in the plane $s p\{\eta, \zeta\}$ and is given by

$$
V=c \eta-c \kappa\left(\tan \theta_{0}\right) \zeta
$$

where $c \in \mathbb{R}_{0}$ and $\theta_{0}=$ constant.

Therefore, we can give the following corollary which gives the relationship between $\xi$-strip slant helices and $\eta$-strip slant helices.

Corollary 11 Let the curve $\alpha$ be a principal curve on $M$ . Then, $(\alpha, M)$ is a $\xi$-strip slant helix if and only if $(\alpha, M)$ is an $\eta$-strip slant helix with the axis (2.29).

Finally, let us characterize $\zeta$-type slant helices.

Case 3 ( $\zeta$-strip slant helices) If $(\alpha, M)$ is a $\zeta$-strip slant helix parameterized by the arclength $s$ in $\mathbb{E}^{3}$, then according to Definition 4 , there exists a nonzero constant vector field $U \in \mathbb{E}^{3}$ such that

$$
g(\zeta, W)=c, \quad c \in \mathbb{R}_{0}
$$

With respect to the Frenet frame $\{\xi, \eta, \zeta\}$ of $(\alpha, M)$, the fixed direction $U$ can be decomposed as

$$
W=w_{1} \xi+w_{2} \eta+c \zeta
$$

where $w_{1}$ and $w_{2}$ are differentiable functions of the curvatures. Differentiating the equation (2.21) with respect to $s$ and using the equations (2.3), we obtain the following system of differential equations

$$
\left\{\begin{aligned}
w_{1}^{\prime}-w_{2} k_{g}-c k_{n} & =0 \\
w_{2}^{\prime}+w_{1} k_{g}-c \tau_{g} & =0 \\
w_{1} k_{n}+u_{2} \tau_{g} & =0
\end{aligned}\right.
$$

From the first and the third equations of(2.32) we get 
CBÜ F Bil. Dergi., Cilt 13, Sayı 1, 2017, 113-123 s

$$
\left\{\begin{array}{l}
w_{1}=c e^{-\int \frac{k_{n} k_{g}}{\tau_{g}} d s}\left(\int k_{n} e^{\int \frac{k_{n} k_{g}}{\tau_{g}} d s} d s\right), \\
w_{2}=-c \frac{k_{n}}{\tau_{g}} e^{-\int \frac{k_{n} k_{g}}{\tau_{g}} d s}\left(\int k_{n} e^{\int \frac{k_{n} k_{g}}{\tau_{g}} d s} d s\right),
\end{array}\right.
$$

where $c \in \mathbb{R}_{0}$. Substituting (2.33) in the second equation of (2.32), we obtain that the curvature functions of $(\alpha, M)$ satisfy the relation

$$
a\left[\left(\frac{k_{n}}{\tau_{g}}\right)^{\prime}-k_{g}^{2}\left(\frac{k_{n}}{\tau_{g}}\right)^{2}-k_{g}\right]+\frac{k_{n}^{2}}{\tau_{g}}+\tau_{g}=0,
$$

where $a=e^{-\int \frac{k_{n} k_{g}}{\tau_{g}} d s}\left(\int k_{n} e^{\int \frac{k_{n} k_{g}}{\tau_{g}} d s} d s\right)$.

Conversely, assume that (2.34) holds. Consider the vector $W$ given by

$$
\begin{aligned}
& W=c e^{-\int \frac{k_{n} k_{g}}{\tau_{g}} d s}\left(\int k_{n} e^{\int \frac{k_{n} k_{g}}{\tau_{g}} d s} d s\right) \xi \\
& -c \frac{k_{n}}{\tau_{g}} e^{-\int \frac{k_{n} k_{g}}{\tau_{g}} d s}\left(\int k_{n} e^{\int \frac{k_{n} k_{g}}{\tau_{g}} d s} d s\right) \eta+c \zeta,
\end{aligned}
$$

where $c \in \mathbb{R}_{0}$. Differentiating the previous equation with respect to $s$ and using the equations (2.3) and (2.32), we find $W^{\prime}=0$. Hence $W$ is a fixed direction. It can be easily checked that

$$
g(\zeta, W)=c, \quad c \in \mathbb{R}_{0}
$$

According to Definition $4,(\alpha, M)$ is a $\zeta$-strip slant helix with the axis $W$.

Therefore, we can give the following theorem and corollary.

Theorem 3 Let $(\alpha, M)$ be a strip in $\mathbb{E}^{3}$ with the curvatures $k_{g}, k_{n}$ and $\tau_{g}$. Then $(\alpha, M)$ is a $\zeta-$ strip slant helix if and only if its curvature functions $k_{g}, k_{n}$. and $\tau_{g}$ satisfy the relation

$$
a\left[\left(\frac{k_{n}}{\tau_{g}}\right)^{\prime}-k_{g}^{2}\left(\frac{k_{n}}{\tau_{g}}\right)^{2}-k_{g}\right]+\frac{k_{n}^{2}}{\tau_{g}}+\tau_{g}=0
$$

where $\tau_{g} \neq 0$ for all $s$ and
CBU J. of Sci., Volume 13, Issue 1, 2017, p 113-123

$$
a=\left(\int k_{n} e^{\int \frac{k_{n} k_{g}}{\tau_{g}} d s} d s\right) e^{-\int \frac{k_{n} k_{g}}{\tau_{g}} d s} .
$$

Corollary 12 The axis of a $\zeta$-strip slant helix $(\alpha, M)$ in $\mathbb{E}^{3}$ is given by

$$
\begin{aligned}
& W=c\left(\int k_{n} e^{\int \frac{k_{n} k_{g}}{\tau_{g}} d s} d s\right) e^{-\int \frac{k_{n} k_{g}}{\tau_{g}} d s} \xi \\
& -c \frac{k_{n}}{\tau_{g}}\left(\int k_{n} e^{\int \frac{k_{n} k_{g}}{\tau_{g}} d s} d s\right) e^{-\int \frac{k_{n} k_{g}}{\tau_{g}} d s} \eta+c \zeta,
\end{aligned}
$$

where $c \in \mathbb{R}_{0}$.

Putting $c=0$ in relation (2.32), we get

$$
\left\{\begin{aligned}
w_{1}^{\prime}-w_{2} k_{g} & =0 \\
w_{2}^{\prime}+w_{1} k_{g} & =0 \\
w_{1} k_{n}+w_{2} \tau_{g} & =0
\end{aligned}\right.
$$

and

$$
\begin{aligned}
& w_{1}=a_{3} e^{-\int \frac{k_{n} k_{g}}{\tau_{g}} d s}, \\
& w_{2}=-a_{3} \frac{k_{n}}{2 \tau_{g}} e^{-\int \frac{k_{n} k_{g}}{\tau_{g}} d s}
\end{aligned}
$$

where $a_{3} \in \mathbb{R}_{0}$.

Therefore, we obtain the next corollary.

Corollary 13 Let $(\alpha, M)$ be a $\zeta$-strip slant helix with the axis $W$ in $\mathbb{E}^{3}$. If its normal vector $\zeta$ is orthogonal to the axis $W$, then the axis $W$ is given by

$$
W=a_{3} e^{-\int \frac{k_{n} k_{g}}{\tau_{g}} d s} \xi-a_{3} \frac{k_{n}}{2 \tau_{g}} e^{-\int \frac{k_{n} k_{g}}{\tau_{g}} d s} \eta
$$

where $a_{3} \in \mathbb{R}_{0}$.

Now, we consider the following special cases when the curvatures $k_{g}, k_{n}$ and $\tau_{g}$ of the strip are zero, respectively.

Case 3.1 Let the curve $\alpha$ is a geodesic curve (i. e. $k_{g}=0$ ), then we find that $\theta=\mp \frac{\pi}{2}$ so by using the definition of $k_{n}$ and $\tau_{g}$, we have $k_{n}=\mp \kappa$ and 


$$
\left\{\begin{aligned}
w_{1}^{\prime}-c k_{n}=0, & \text { From the third equation of }(2.37) \text { we get } \tau_{g}=0 \text { or } \\
w_{2}^{\prime}-c \tau_{g}=0, & u_{2}=0 . \\
w_{1} k_{n}+w_{2} \tau_{g}=0 . &
\end{aligned}\right.
$$

From the first and second equations of (2.35) we get

$$
\begin{aligned}
& w_{1}=c \int k_{n} d s=\mp c \int \kappa d s, \\
& w_{2}=c \int \tau_{g} d s=c \int \tau d s .
\end{aligned}
$$

Substituting (2.36) in the third equation of (2.35), we obtain that the curvature functions of $(\alpha, M)$ satisfy the relation as follows

(i) If $\tau_{g}=0$ for all $s$, then $k_{g}= \pm \kappa$ and $\tau=0$. So from (2.37) we get

$$
\begin{aligned}
& w_{1}=r \cos v, \\
& w_{2}=r \sin v,
\end{aligned}
$$

where $r \in \mathbb{R}^{+}$and $v=\mp \int \kappa d s$. Therefore, the axis of $(\alpha, M)$ is given by

$$
W=r \cos (v) \xi+r \sin (v) \eta+c \zeta
$$

$$
k_{g} \int k_{g} d s+\tau_{g} \int \tau_{g} d s=0
$$

where $c \in \mathbb{R}_{0}$.

or

$$
\kappa \int \kappa d s+\tau \int \tau d s=0 .
$$

(ii) If $w_{2}=0$ for all $s$, then from (2.37) we have

$$
w_{1}=c \frac{\tau_{g}}{k_{g}}=\text { constant }
$$

Thus, we have the frame $\{\xi, \eta, \zeta\}$ of the strip $(\alpha, M)$ as follows:

$$
\left[\begin{array}{l}
\xi^{\prime}(s) \\
\eta^{\prime}(s) \\
\zeta^{\prime}(s)
\end{array}\right]=\left[\begin{array}{ccc}
0 & 0 & \mp \kappa \\
0 & 0 & \tau \\
\pm \kappa & -\tau & 0
\end{array}\right]\left[\begin{array}{c}
\xi(s) \\
\eta(s) \\
\zeta(s)
\end{array}\right]
$$

and the axis of $(\alpha, M)$ always lies in the plane $s p\{\eta, \zeta\}$ and is given by

$$
W=w_{1} \xi+w_{2} \eta+c \zeta
$$

where $c \in \mathbb{R}_{0}$ and

$$
\begin{aligned}
& w_{1}=\mp c \int \kappa d s \\
& w_{2}=c \int \tau d s .
\end{aligned}
$$

Also, by using the definition of $k_{g}$ and $\tau_{g}$, we have $k_{g}=\mp \kappa$ and $\tau_{g}=\tau$ since $k_{n}=0$. Then

$$
w_{1}=\mp c \frac{\tau}{\kappa},
$$

and the axis of $(\alpha, M)$ is

$$
U=\mp c \frac{\tau}{\kappa} \xi+c \zeta,
$$

where $\frac{\tau}{\kappa}=$ constant and $c \in \mathbb{R}_{0}$.

Therefore, we can give the following corollaries.

Corollary 14 Let $(\alpha, M)$ be a $\zeta$-strip slant helix with the axis $W$ is given by (2.39). If the curve $\alpha$ of $(\alpha, M)$ is an asymptotic curve on $M$, then the curve $\alpha$ is a

Case 3.2 Let the curve $\alpha$ is an asymptotic curve (i. general helix.

e. $\left.k_{n}=0\right)$, then from (2.32) we have

$$
\left\{\begin{aligned}
w_{1}^{\prime}-w_{2} k_{g} & =0 \\
w_{2}^{\prime}+w_{1} k_{g}-c \tau_{g} & =0 \\
w_{2} \tau_{g} & =0
\end{aligned}\right.
$$

Also, we can give the following corollary which gives the relationship between $\xi$-strip slant helices and $\zeta$-strip slant helices.

Corollary 15 Let the curve $\alpha$ be an asymptotic curve on 
CBÜ F Bil. Dergi., Cilt 13, Sayı 1, 2017, 113-123 s

$M$. Then, $(\alpha, M)$ is a $\xi$-strip slant helix if and only if $(\alpha, M)$ is a $\zeta$-strip slant helix with the axis (2.39).

Case 3.3 Let the curve $\alpha$ is a principal curve (i. e. $\left.\tau_{g}=0\right)$, then from (2.32) we have

$$
\left\{\begin{array}{r}
w_{1}^{\prime}-w_{2} k_{g}-c k_{n}=0 \\
w_{2}^{\prime}+w_{1} k_{g}=0 \\
w_{1} k_{n}=0
\end{array}\right.
$$

From the third equation of (2.40) we get $k_{n}=0$ or $w_{1}=0$.

(i) If $k_{n}=0$ for all $s$, then $k_{g}= \pm \kappa$ and $\tau=0$. So from (2.40) we get

$$
\begin{aligned}
& w_{1}=R \cos \varphi, \\
& w_{2}=R \sin \varphi,
\end{aligned}
$$

where $R \in \mathbb{R}^{+}$and $\varphi=\mp \int \kappa d s$. Therefore, the axis of $(\alpha, M)$ is given by

$$
W=R \cos (\varphi) \xi+R \sin (\varphi) \eta+c \zeta
$$

where $c \in \mathbb{R}_{0}$.

(ii) If $w_{1}=0$ for all $s$, then from (2.40) we have

$$
w_{2}=-c \frac{k_{n}}{k_{g}}=\text { constant }
$$

Also, by using the definition of $\tau_{g}$ we have $\theta=\int \tau d s$. Thus, we obtain $k_{n}=\kappa \sin \left(\int \tau d s\right)$, $k_{g}=\kappa \cos \left(\int \tau d s\right)$ and the axis of $(\alpha, M)$ is

$$
W=-c \frac{k_{n}}{k_{g}} \eta+c \zeta,
$$

where $c \in \mathbb{R}_{0}$.

Therefore, we can give the following corollary which gives the relationship between $\eta$-strip slant helices and $\zeta$-strip slant helices.

Corollary 16 Let the curve $\alpha$ of $(\alpha, M)$ be an asymptotic curve on $M$. Then, $(\alpha, M)$ is an $\eta$-strip slant helix with (2.24) if and only if $(\alpha, M)$ is a $\zeta$-strip
CBU J. of Sci., Volume 13, Issue 1, 2017, p 113-123

slant helix with the axis (2.41).

\section{References}

[1] Struik, D. J. Lectures on classical differential geometry. Addison Wesley, Dover, 1988; 240 pp.

[2] Izumiya, S.; Tkeuchi, N. New special curves and developable surfaces. Turk J. Math. 2004; 28, 153-163.

[3] Kula, L.; Yayli, Y. On slant helix and its spherical indicatrix. Appl. Math. Comput. 2005; 169(1), 600-607.

[4] Kula, L.; Ekmekci, N.; Yayli, Y.; Ilarslan, K. Characterizations of slant helices in Euclidean 3-space. Turk. J. Math. 2010; 34, 261-273.

[5] Ali, A. T.; López, R. Slant helices in Euclidean 4-space $E^{4}$. J. Egyptian Math. Soc. 2010; 18(2), 223-230.

[6] Ali, A. T.; Turgut, M. Some characterizations of slant helices in the Euclidean space $\mathbb{E}^{n}$. Hacet. J. Math. Stat. 2010; 39(3), 327-336.

[7] Hacisalihoglu, H. H. On the relations between the higher curvatures of a curve and a strip. Communications de la Faculté des Sciences de L'Université d' Ankara Serie A1. 1982; 31, 5-14.

[8] Sabuncuoglu, A.; Hacisalihoglu, H. H. Higher curvatures of a strip. Comm. Fac. Sci. Univ. Ankara Sér. A1 Math. 1975; 24, 25-33. 